University of Nebraska - Lincoln

DigitalCommons@University of Nebraska - Lincoln

Xia Hong Publications

Research Papers in Physics and Astronomy

2007

\title{
Anisotropic magnetoresistance in colossal magnetoresistive La1-xSrxMnO3 thin films
}

Jeng-Bang Yau

X. Hong

A. Posadas

C. H. Ahn

W. Gao

See next page for additional authors

Follow this and additional works at: https://digitalcommons.unl.edu/physicshong

Part of the Atomic, Molecular and Optical Physics Commons, and the Engineering Physics Commons

This Article is brought to you for free and open access by the Research Papers in Physics and Astronomy at DigitalCommons@University of Nebraska Lincoln. It has been accepted for inclusion in Xia Hong Publications by an authorized administrator of DigitalCommons@University of Nebraska Lincoln. 
Authors

Jeng-Bang Yau, X. Hong, A. Posadas, C. H. Ahn, W. Gao, E. Altman, Y. Bason, L. Klein, M. Sidorov, and Z. Krivokapic 


\title{
Anisotropic magnetoresistance in colossal magnetoresistive $\mathrm{La}_{1-x} \mathrm{Sr}_{x} \mathrm{MnO}_{3}$ thin films
}

\author{
Jeng-Bang Yau, ${ }^{\text {a) }}$ X. Hong, A. Posadas, and C. H. Ahn \\ Department of Applied Physics, Yale University, New Haven, Connecticut 06520, USA \\ W. Gao and E. Altman \\ Department of Chemical Engineering, Yale University, New Haven, Connecticut 06520, USA \\ Y. Bason and L. Klein \\ Department of Physics, Bar Ilan University, Ramat Gan 52900, Israel
}

M. Sidorov and Z. Krivokapic

Advanced Micro Devices, P.O. Box 3453, Sunnyvale, California 94088, USA

(Received 30 May 2007; accepted 19 September 2007; published online 16 November 2007)

\begin{abstract}
We report on magnetic field and temperature-dependent measurements of the anisotropic magnetoresistance (AMR) in epitaxial $\mathrm{La}_{1-x} \mathrm{Sr}_{x} \mathrm{MnO}_{3}$ (LSMO) thin films. While in $3 d$ ferromagnetic alloys increasing the magnetization, either by reducing the temperature or increasing the magnetic field, increases the AMR, we find that in LSMO films the AMR dependence on magnetization displays nonmonotonic behavior which becomes particularly pronounced in lightly doped compounds. We believe that this behavior is related to the inhomogeneity exhibited by these materials. () 2007 American Institute of Physics. [DOI: 10.1063/1.2811919]
\end{abstract}

\section{INTRODUCTION}

Since the observation of colossal magnetoresistance (CMR) (Ref. 1) in doped manganites, these materials have been extensively studied, in particular for their correlation of structural, magnetic, electronic, and orbital degrees of freedom. Numerous experiments ${ }^{2}$ have investigated the magnetotransport properties of these materials, including their anisotropic magnetoresistance (AMR), which is the difference in resistance determined by the relative orientation between the magnetization and the current density. AMR has been an effect of practical and theoretical interest since its discovery by Thomson. ${ }^{3}$ For example, the AMR effect in transition metal thin films has been utilized to fabricate magnetic devices such as magnetic read heads and sensors. ${ }^{4}$ Furthermore, recent discovery of the giant planar Hall effect (GPHE), ${ }^{5}$ which is a phenomenon originating from the AMR effect in CMR manganites, is deemed promising for nonvolatile storage devices such as magnetic random access memory (MRAM). ${ }^{6}$ In addition, studying AMR sheds light on how it links to other fundamental properties, such as magnetocrystalline anisotropy and spin-orbit coupling in magnetic materials. ${ }^{7,8}$

AMR in ferromagnetic alloys originates from the spinorbit coupling that yields the dependence of the scattering rate of the $s$ band conduction electrons on the angle between their current direction and the spin direction of the localized $3 d$ electrons. ${ }^{9}$ Mott ${ }^{10}$ developed a two-current model to explain the electrical conductivity in transition metals, considering the effect caused by the interplay between the exchange-split bands. Based on Mott's model, subsequent theoretical work has been successful in accounting for the AMR measured in $3 d$ ferromagnetic alloys: ${ }^{11-13}$ the magni-

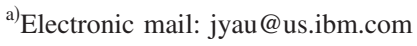

tude of the AMR approaches zero above the Curie temperature and increases approximately linearly with decreasing temperatures below $T_{c}$, saturating at low temperatures. In addition, at low magnetic fields, the AMR exhibits a monotonic field-induced change that saturates at high fields. ${ }^{14,15}$

Recent studies of AMR measured in $\mathrm{La}_{1-x} \mathrm{Ca}_{x} \mathrm{MnO}_{3}$ (LCMO) thin films reported a nonmonotonic temperature dependence, ${ }^{16-22}$ different from what is observed in the AMR measured in $3 d$ transition metals, suggesting that different models are required. However, before such models can be formulated, a more comprehensive picture of the AMR behavior in CMR manganites is needed. In this work we conducted magnetotransport measurements on epitaxial $\mathrm{La}_{1-x} \mathrm{Sr}_{x} \mathrm{MnO}_{3}$ thin films as a function of temperature, magnetic field, and doping concentration. We find that the AMR is nonmonotonic as a function of temperature or field, particularly in lightly doped samples. We suggest that this behavior is related to local electronic nonuniformity, which is more pronounced in compounds near the metal-insulator phase transition. According to this scenario, the nonuniformity enhances spin-dependent scattering which leads to increased AMR. Consequently, increasing magnetization, either by reducing temperature or increasing field, yields two competing effects. On the one hand, increased magnetization enhances spin polarization, leading to increased AMR. On the other hand, increased magnetization suppresses the nonuniformity, thus decreasing the spin-dependent scattering and the AMR.

\section{EXPERIMENT}

The starting materials for our experiment are $400 \AA$ thick $\mathrm{La}_{1-x} \mathrm{Sr}_{x} \mathrm{MnO}_{3}$ films of various doping concentrations $(x=0.16,0.20,0.33$, and 0.35$)$ that were epitaxially deposited on single-crystal $\mathrm{SrTiO}_{3}(001)$ substrates by off-axis magne- 

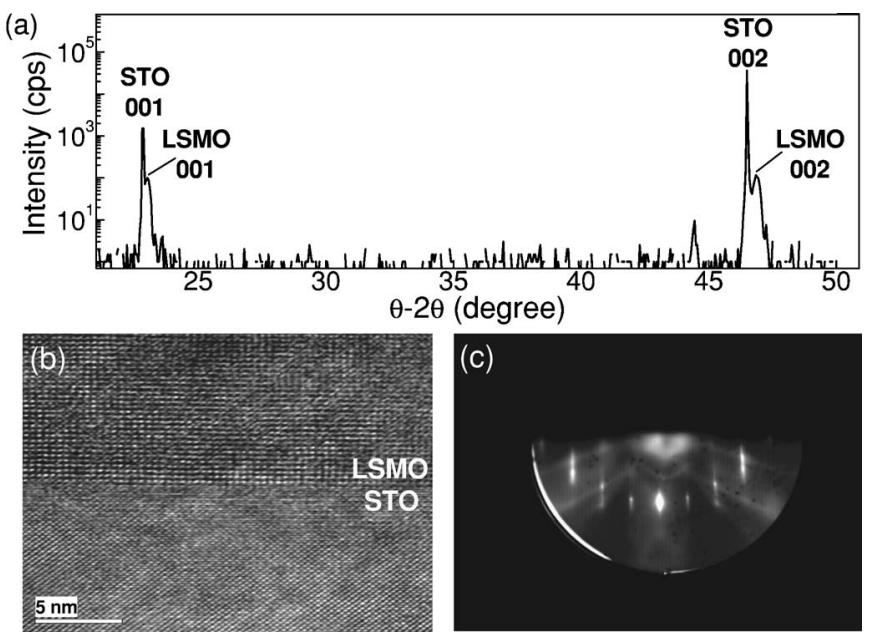

FIG. 1. (a) $\theta-2 \theta$ x-ray diffraction scan taken on a $400 \AA$ thick LSMO film. The LSMO peaks are the shoulders of the STO substrate peaks. (b) Highresolution TEM image of a LSMO film. (c) RHEED image of a LSMO film.

tron sputtering. $\theta-2 \theta$ x-ray diffraction (XRD) [Fig. 1(a)] shows $c$-axis oriented growth, with no signs of impurity phases. Off-axis scans conducted on the same films give an in-plane lattice constant of $\sim 3.88 \AA$, revealing relaxation of the films. The film surfaces were characterized by atomic force microscopy, which shows a typical root-mean-square surface roughness of $\sim 2 \AA$. Figure 1(b), a high-resolution transmission electron microscopy (TEM) image, indicates good film quality, without signs of grain boundaries or other structural defects. In addition, reflection high-energy electron diffraction (RHEED) was carried out on a LSMO film in a separate chamber equipped with a differentially pumped 15 $\mathrm{keV}$ electron gun and a fluorescent screen mounted on the opposite side of the chamber. The film was cleaned by oxygen plasma before the RHEED measurement. Auger electron spectroscopy (AES) confirmed that the surface was free of impurities. Figure 1(c) shows RHEED taken along the [100] direction of the film. The parallel streaked pattern suggests epitaxial growth of the film with a flat surface. Depending on $x$, the magnetic Curie temperature $T_{c}$ of these samples ranges from $\sim 200$ to $\sim 350 \mathrm{~K}$. Figure 2 shows the magnetization and resistivity measured as a function of temperature on an $x=0.16 \mathrm{LSMO}$ film. The resistivity peaks at $\sim 240 \mathrm{~K}$, below

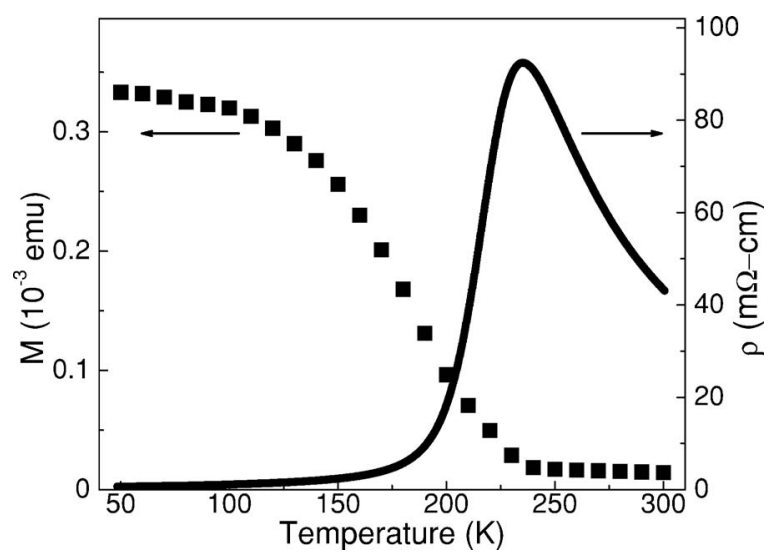

FIG. 2. Magnetization and resistivity data measured on $\mathrm{La}_{0.84} \mathrm{Sr}_{0.16} \mathrm{MnO}_{3}$ as a function of temperature. (a)

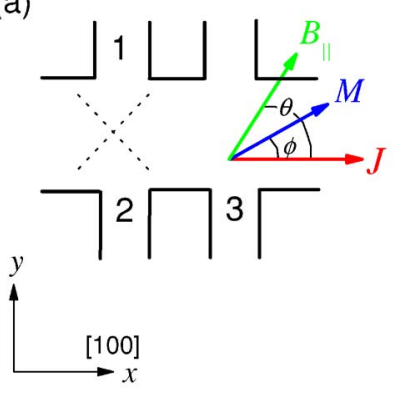

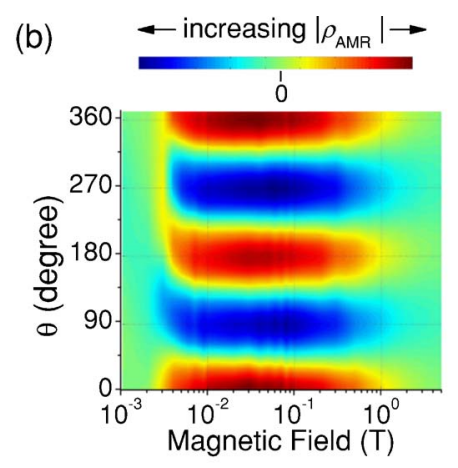

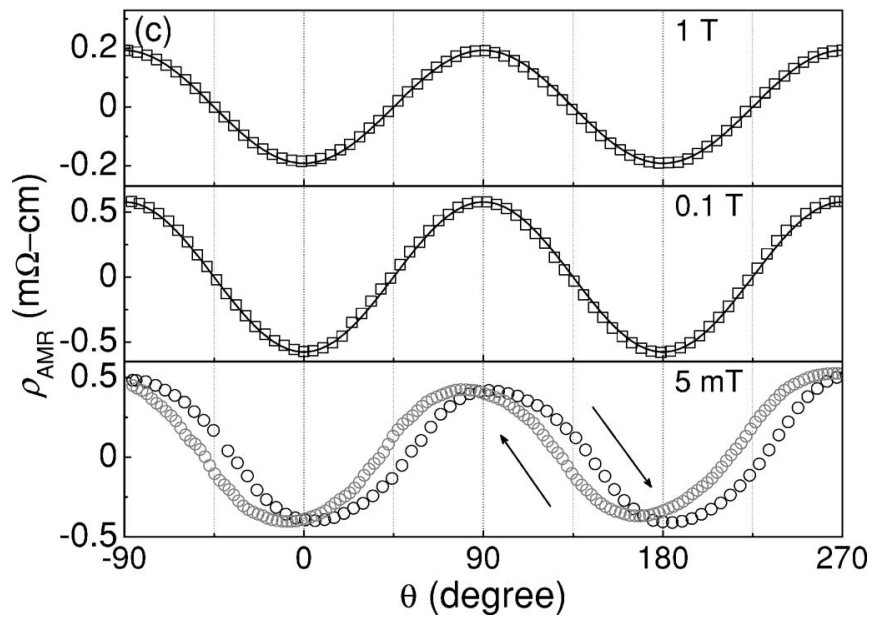

FIG. 3. (Color online) (a) Schematic of the pattern used for magnetotransport measurements of the AMR. (b) Longitudinal resistivity $\rho_{\mathrm{AMR}}$ measured as a function of applied in-plane magnetic field and angle $\theta$ at $T=200 \mathrm{~K}$ in $\mathrm{La}_{0.84} \mathrm{Sr}_{0.16} \mathrm{MnO}_{3}$. (c) $\rho_{\text {AMR }}$ vs $\theta$ at $T=200 \mathrm{~K}$ in $\mathrm{La}_{0.84} \mathrm{Sr}_{0.16} \mathrm{MnO}_{3}$ with different in-plane magnetic fields. The lines are fits to $\cos ^{2} \theta$.

which it exhibits a steep decrease, in accord with the onset of ferromagnetic ordering. The samples were patterned for magnetotransport measurements [shown schematically in Fig. 3(a)], with the current path aligned along either the [100] or [010] direction of the films.

\section{RESULTS AND DISCUSSION}

The electric field induced within a single domain ferromagnetic film with in-plane magnetization and a uniformly distributed current density $j$ in the $x$ direction is described by

$$
E_{x}=\rho_{\perp} j_{x}+\left(\rho_{\|}-\rho_{\perp}\right) j_{x} \cos ^{2} \phi=\rho_{\perp} j_{x}+\rho_{\mathrm{AMR}} j_{x},
$$

where $\phi$ is the angle between $j$ and the magnetization, and $\rho_{\|}$ and $\rho_{\perp}$ are the resistivities for current oriented parallel and perpendicular to the magnetization, respectively. The AMR effect is characterized by $\rho_{\mathrm{AMR}}=\left(\rho_{\|}-\rho_{\perp}\right) \cos ^{2} \phi$ $=\Delta \rho_{\text {AMR }} \cos ^{2} \phi$. We first investigated the AMR in $\mathrm{La}_{0.84} \mathrm{Sr}_{0.16} \mathrm{MnO}_{3}$, which is characterized by a resistivitypeak temperature $\left(T_{p}\right)$ of $\sim 240 \mathrm{~K}$, as shown in Fig. 2. $\rho_{\mathrm{AMR}}$ is measured between contacts 2 and 3 as shown in Fig. 3(a) as a function of both the in-plane magnetic field $\left(B_{\|}\right)$and $\theta$, the angle between the current and the magnetic field. ${ }^{23}$ The result, summarized in Fig. 3(b), provides a panoramic scope and quantitative determination of the AMR. $\rho_{\mathrm{AMR}}$ was measured as a function of $\theta$ at 36 different in-plane magnetic fields ranging from $10 \mathrm{mT}$ to $5 \mathrm{~T}$. In this field range, good agreement with Eq. (1) is found. At lower fields, higher order 


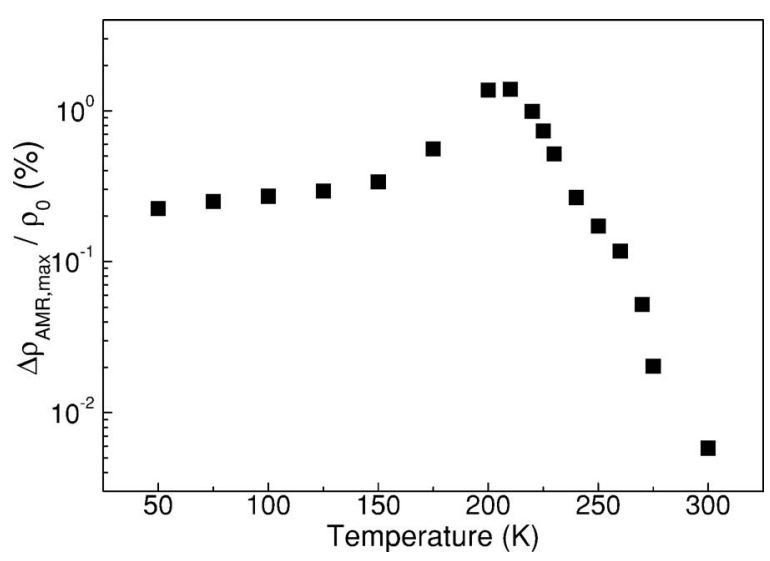

FIG. 4. Temperature dependence of $\Delta \rho_{\mathrm{AMR}, \max } / \rho_{0}$ measured in $\mathrm{La}_{0.84} \mathrm{Sr}_{0.16} \mathrm{MnO}_{3}$.

terms induced by the magnetocrystalline anisotropy may be needed. $^{24}$

It can be seen in Fig. 3(b) that the amplitude of $\rho_{\mathrm{AMR}}$ appears to change with the in-plane magnetic field. This magnetic field dependence is further illustrated in Fig. 3(c), in which $\rho_{\text {AMR }}$ traces taken at different magnetic fields are plotted. ${ }^{25} \rho_{\text {AMR }}$ shows a $\cos ^{2} \theta$ dependence at relatively high magnetic fields $(0.1$ and $1 \mathrm{~T})$, while at a much lower field $(5 \mathrm{mT}), \rho_{\mathrm{AMR}}$ deviates from the expected $\cos ^{2} \theta$ dependence. The latter is caused by the biaxial magnetic anisotropy, ${ }^{5}$ which becomes significant and manifests itself as hysteresis in field directions, as can be seen in both Figs. $3(\mathrm{~b})$ and $3(\mathrm{c}){ }^{26}$

The plot shown in Fig. 3(b) allows us to locate $\Delta \rho_{\mathrm{AMR}, \max }$, which is the maximum $\Delta \rho_{\mathrm{AMR}}$ at a given temperature. We then studied the temperature dependence of the magnitude of the AMR by repeating the same field-angle magnetotransport measurement shown in Fig. 3(b) at various temperatures. According to Eq. (1), $\Delta \rho_{\mathrm{AMR}, \max }$ is obtained by selecting the $\rho_{\text {AMR }}$ that has the maximum amplitude in its $\cos ^{2} \theta$ angular dependence curve at different temperatures; these results are plotted in Fig. 4 . We find that $\Delta \rho_{\text {AMR,max }}$ is temperature dependent, with its value reaching a maximum at approximately $T \sim 210 \mathrm{~K}$. One striking feature we observe in Fig. 4 is that $\Delta \rho_{\text {AMR,max }}$ decreases as the temperature decreases. This behavior, which has been observed in AMR measured in other manganite thin films, ${ }^{16-22}$ differs from what is observed in $3 d$ ferromagnetic metals, where $\Delta \rho_{\mathrm{AMR}}$ increases with decreasing temperature. ${ }^{14,27}$

To analyze the data obtained from the above magnetotransport measurements in more detail, in Fig. 5(a) we plot the relative AMR ratio, defined as $\Delta \rho_{\mathrm{AMR}} / \rho_{0}$, as a function of the magnetic field and temperature. A similar nonmonotonic temperature dependence is observed for $\Delta \rho_{\mathrm{AMR}} / \rho_{0}$ over a wide range of applied magnetic fields. Moreover, at temperatures in the vicinity of $T_{p}$, we discover that $\Delta \rho_{\mathrm{AMR}} / \rho_{0}$ at $T \lesssim T_{p}$ exhibits a different field dependence as the temperature changes. This unusual feature is further illustrated in Fig. 5(b). At $T=150 \mathrm{~K}, \Delta \rho_{\mathrm{AMR}} / \rho_{0}$ remains nearly constant throughout the range of applied magnetic fields. For $\Delta \rho_{\mathrm{AMR}} / \rho_{0}$ measured at $T=250 \mathrm{~K}$, it remains nearly constant for fields up to $\sim 0.2 \mathrm{~T}$ and starts to increase
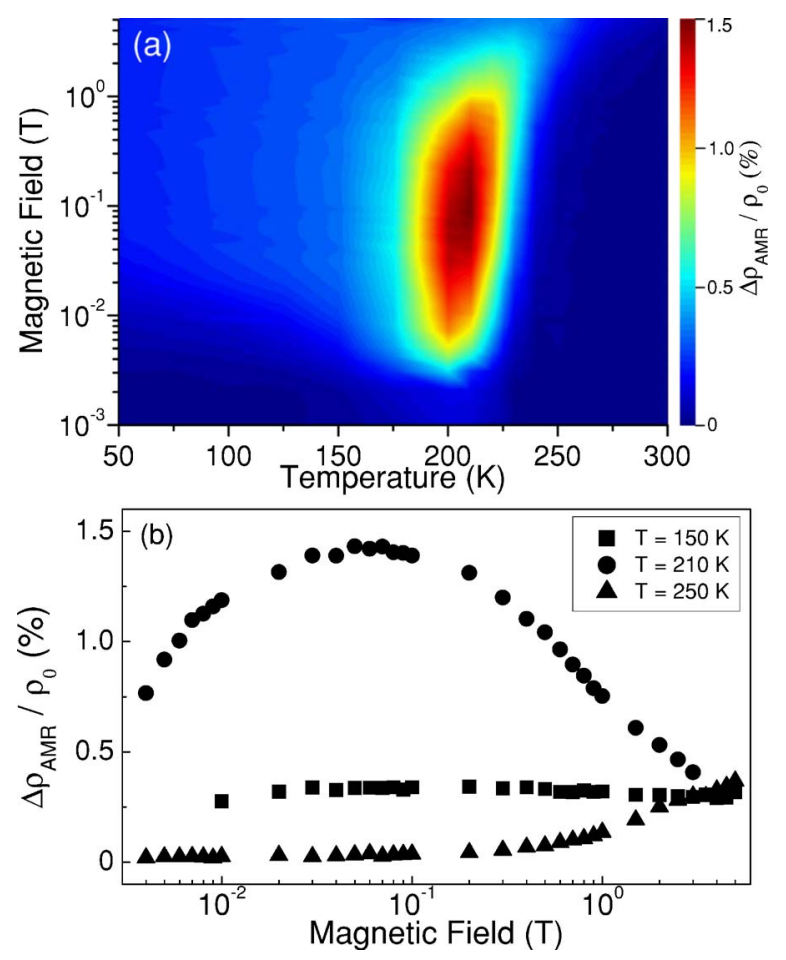

FIG. 5. (Color online) (a) $\Delta \rho_{\mathrm{AMR}} / \rho_{0}$ measured as a function of temperature and magnetic field in $x=0.16 \mathrm{LSMO}$. (b) Field dependence of $\Delta \rho_{\mathrm{AMR}} / \rho_{0}$ for $x=0.16 \mathrm{LSMO}$ measured at various temperatures.

with field as the magnetization aligns itself with the field. $\Delta \rho_{\text {AMR }} / \rho_{0}$ measured at $T=210 \mathrm{~K}$, however, exhibits a different field dependence, with a maximum occurring at $\sim 0.09 \mathrm{~T}$. At fields below $0.09 \mathrm{~T}, \Delta \rho_{\mathrm{AMR}} / \rho_{0}$ increases with field, which would be expected in view of the increasing magnetization. Surprisingly, at fields above $0.09 \mathrm{~T}$, $\Delta \rho_{\mathrm{AMR}} / \rho_{0}$ decreases as the field increases.

In order to understand the unusual temperature and field dependence, shown in Figs. 5(a) and 5(b), respectively, we first consider extrinsic effects such as grain boundaries or twinning. In general, as the film thickness increases, the epitaxial strain is relaxed, leading to the occurrence of twinning and the development of grainy microstructures in the film. While our LSMO films are relaxed according to the XRD data [Fig. 1(a)], the low resistivity at low temperatures as shown in Fig. 2 indicates small contribution of scattering by microstructures, suggesting negligible effects of grain boundaries. ${ }^{28,29}$ In addition, the decreasing AMR with decreasing temperature, as shown in Fig. 5, demonstrates good crystalline quality in our $x=0.16$ LSMO. $^{20}$ To address the effect of twinning, it is worth noting that several previous works have reported the occurrence of twinning in LSMO thin films grown by pulsed-laser deposition on STO(001) substrates. ${ }^{30}$ The twin patterns observed in these films have been explained as a result of strain relaxation within the distorted rhombohedral LSMO crystal lattice, ${ }^{31}$ resulting in the formation of magnetic anisotropy. ${ }^{32,33}$ Such anisotropy is believed to account for the magnetization behavior and magnetoresistive properties in LSMO thin films at low magnetic fields. The AMR measurements on our LSMO films, however, were conducted at relatively high magnetic fields. The magnetization is always oriented along the field direction 


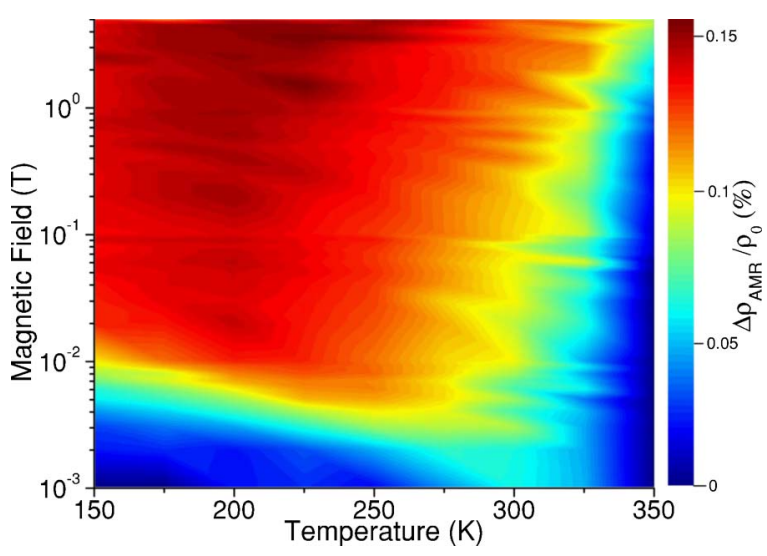

FIG. 6. (Color online) $\Delta \rho_{\mathrm{AMR}} / \rho_{0}$ measured as a function of temperature and magnetic field in $x=0.35$ LSMO.

throughout the measurement, provided that the applied magnetic field is large enough to overcome the magnetic anisotropy and fully magnetize the film. Considering that the data shown in Figs. 5(a) and 5(b) were obtained by measuring the AMR at magnetic fields where the influence of the in-plane biaxial magnetic anisotropy is negligible, the existence of twin boundaries would not affect the AMR and hence does not underlie the unusual field and temperature dependence of the AMR.

Here, we provide an explanation that considers the role of the nominal chemical doping concentration $(x=0.16)$, which is near the ferromagnetic percolation threshold $x_{c}$ $\approx 0.15,{ }^{34}$ the location of the metal-insulator phase boundary. At this composition, different electronic and magnetic phases may coexist. In this case, the applied magnetic field yields two different effects: (a) it increases the local magnetization; and (b) it makes the film more uniform through growing magnetic homogeneity. Increasing the local magnetization should increase the AMR; however, increasing film uniformity could lead to a decrease of spin-dependent scattering and hence decrease the AMR. ${ }^{35}$ Such opposite influences may be the source of the nonmonotonic field dependence observed in the most electronically inhomogeneous films. By similar reasoning, the nonmonotonic temperature dependence of $\Delta \rho_{\mathrm{AMR}} / \rho_{0}$ may be understood as follows. As the temperature decreases, the magnetization increases, which is expected to yield a larger AMR. On the other hand, an increase of magnetization in these thin films is accompanied by a greater uniformity, which may decrease the spin-dependent scattering. Again, these two competing effects would yield the observed behavior as illustrated in Fig. 5(a). This interpretation is corroborated by the data shown in Fig. 6, which is the AMR measured in an $x=0.35$ LSMO thin film that is more electronically homogeneous, with a $T_{c} \gtrsim 350 \mathrm{~K}$. It is clear from Fig. 6 that the AMR measured in the heavily doped LSMO film exhibits an essentially monotonic dependence on the magnetic field and temperature. The slight deviation of the temperature dependence from monotonicity for magnetic fields below $\sim 0.01 \mathrm{~T}$ is due to the fact that the AMR measurement is influenced by the biaxial magnetic anisotropy, which becomes significant at low magnetic fields.

Next, we consider whether we could apply the above picture to explain the similar nonmonotonic temperature dependence of the AMR observed in LCMO. ${ }^{16-22}$ These works recognize spin-orbit interaction to be the basic mechanism for the AMR effect in manganites, and present various simple models accordingly to address the unusual temperature dependence of the AMR observed in LCMO. These models include: spin-orbit coupling for carriers in $d$-bands and some detailed features in the band structure, ${ }^{16,20}$ local orbit distortion induced by spin-orbit interactions that affect local hopping conduction processes near $T_{p},{ }^{18}$ nonidentical magnetic properties at various parts of the film caused by an inhomogeneous crystal structure, ${ }^{21}$ and an anisotropic Fermi surface for the carriers. ${ }^{22}$ While the pictures described by these models are plausible, no further experimental or theoretical details were provided to elaborate on how one can connect these models with the unusual temperature dependence of the AMR.

The AMR measurements reported in Refs. 16-22 were carried out on $x \sim 0.3$ LCMO thin films, which were discovered to exhibit phase-separated phenomena that are due to inhomogeneous structures of metallic (ferromagnetic) and insulating (paramagnetic) areas in the films. ${ }^{36}$ It is suggested in Ref. 34 that the phase separation observed in $x \sim 0.3$ LCMO below $T_{c}$ is magnetization dependent, with the ferromagnetic metallic phase percolating in response to increasing magnetic fields, leading to an enhanced conductivity, while above $T_{c}$ the insulating paramagnetic phase dominates, resulting in a decreased conductivity. The percolation of the ferromagnetic metallic phase leads to increased magnetization, which should increase the AMR. At the same time the film becomes more uniform, suggesting less spin-dependent scattering and hence reduced AMR. As described previously, these two competing effects may yield the nonmonotonic AMR as a function of magnetic field. One should expect that decreasing the temperature may affect the AMR in a similar manner. Thus, the scenario of competing effects, which is applied to interpret the nonmonotonic temperature and magnetic field dependence of the AMR in LSMO, should also be applicable in the explanation for the similar behavior observed in their Ca-doped counterpart.

Finally, to further investigate the dissimilar AMR effects observed in differently doped LSMO thin films as shown in Figs. 5(a) and 6], we examined the doping dependence of the AMR by measuring $\Delta \rho_{\text {AMR }} / \rho_{0}$ in LSMO thin films with various $x$ values as a function of temperature at $B_{\|}=0.7 \mathrm{~T}$. In Fig. 7 we plot $\Delta \rho_{\text {AMR }} / \rho_{0}$ versus $T$ for $x=0.16,0.20,0.33$, and 0.35. Several features are observed. As shown in Fig. 7, the relative AMR ratio exhibits a nonmonotonic behavior, similar to what is observed in Fig. 5. Even when the system becomes more metallic as $x$ increases, the AMR measured in LSMO thin films shows behavior different from that observed in $3 d$ ferromagnetic metals. In addition, the temperature where the peak in each $\Delta \rho_{\mathrm{AMR}} / \rho_{0}$ curve occurs appears to increase with $x$. The increase may be an effect of the magnetization on the AMR. Also, $\Delta \rho_{\mathrm{AMR}} / \rho_{0}$ decreases substantially with $x$. The observed difference in AMR between the differently doped films may be related to the structural changes that are induced by increased doping, affecting the spin-orbit coupling. Changes of $x$ in crystals of LSMO affect 


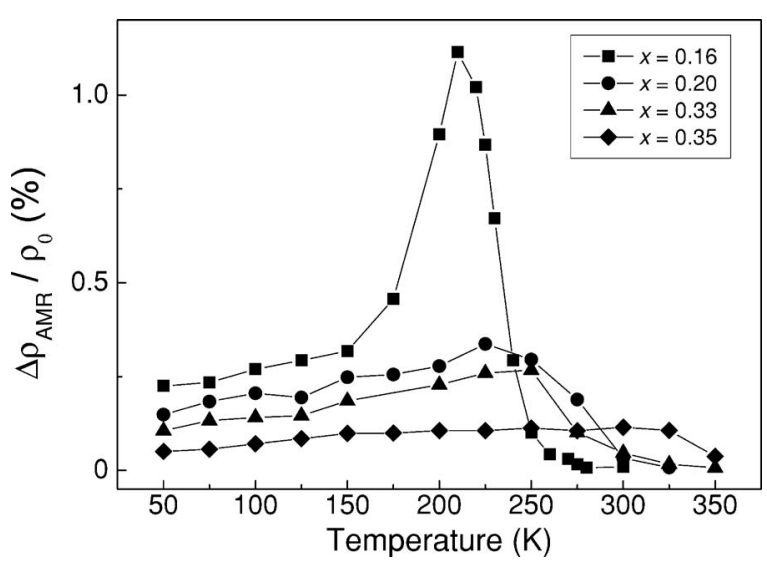

FIG. 7. $\Delta \rho_{\mathrm{AMR}} / \rho_{0}$ vs $T$ for LSMO thin films with different doping concentrations. Data are measured in a $0.7 \mathrm{~T}$ magnetic field. The lines are a guide to the eye.

the coupling between the $e_{g}$ conduction electrons and the $t_{2 g}$ local spins, and an increase of $x$ drives the system effectively toward the weak coupling regime. ${ }^{37}$ The reduction of spinorbit coupling could be realized through relaxed lattice distortion associated with the aliovalent substitution of $\mathrm{La}$ by $\mathrm{Sr}$ in LSMO. Increasing $x$ results in a smaller lattice distortion and hence less scattering of the conduction electrons, translating into a diminished effect on the AMR. ${ }^{18,22,38}$ This can explain reduced AMR but not the qualitative change from nonmonotonic to monotonic behavior as a function of temperature and field. Moreover, that $\Delta \rho_{\mathrm{AMR}} / \rho_{0}$ for $x=0.16 \mathrm{ex}-$ hibits a peak value significantly larger than those for other compositions may be due to the aforementioned coexistence of various phases in the $x=0.16$ sample. ${ }^{39}$

\section{SUMMARY AND CONCLUSIONS}

The AMR in LSMO thin films is nonmonotonic, in contrast to AMR in $3 d$ ferromagnetic metals. We suggest that the temperature, field, and doping dependence measurements of the AMR in LSMO films presented here indicate that, in addition to the magnitude of magnetization and the lattice distortion, the AMR depends on local uniformity. Nonuniformity increases the AMR by increasing the spin-dependent scattering. Therefore, increased magnetization has two competing effects: in addition to increasing spin polarization (which increases the AMR), it increases uniformity (which decreases the AMR). As a function of temperature, this scenario explains the doping dependence, since the more intrinsically nonuniform the samples are, the more pronounced the nonmonotonic behavior is. As a function of field, the scenario also explains the different field dependence observed at various temperatures.

\section{ACKNOWLEDGMENTS}

C.A. and E.A. acknowledge primary support from NSFMRSEC DMR-0520495, and C.A. acknowledges support from ONR. L.K. acknowledges support from the Israel Science Foundation founded by the Israel Academy of Sciences and Humanities. C.A. and L.K. acknowledge support from Grant No. 2002384 from the United States-Israel Binational Science Foundation, Jerusalem, Israel. The color plots shown in Figs. 5 and 6 were created using the MATLAB program written by Y.P. Shkolnikov at Princeton University.

${ }^{1}$ S. Jin, T. H. Tiefel, M. McCormack, R. A. Fastnacht, R. Ramesh, and L. H. Chen, Science 264, 413 (1994).

${ }^{2}$ Colossal Magnetoresistive Oxides, edited by Y. Tokura (Gordon and Breach, Amsterdam, The Netherlands, 2000); J. Z. Sun and A. Gupta, Annu. Rev. Mater. Sci. 28, 45 (1998).

${ }^{3}$ W. Thomson, Proc. R. Soc. London 8, 546 (1856).

${ }^{4}$ Magnetism II - Materials and Applications, edited by É. du Trémolet de Lacheisserie, D. Gignoux, and M. Schlenker (Kluwer, Norwell, MA, 2002).

${ }^{5}$ Y. Bason, L. Klein, J.-B. Yau, X. Hong, and C. H. Ahn, Appl. Phys. Lett. 84, 2593 (2004).

${ }^{6}$ Y. Bason, L. Klein, J. -B. Yau, X. Hong, J. Hoffman, and C. H. Ahn, J. Appl. Phys. 99, 08R701 (2006).

${ }^{7}$ E. D. Dahlberg, R. Riggs, and G. A. Printz, J. Appl. Phys. 63, 4270 (1988). ${ }^{8}$ Th. G. S. M. Rijks, R. Coehoorn, and M. J. M. de Jong, Phys. Rev. B 51, 283 (1995).

${ }^{9}$ J. Smit, Physica, 17, 610 (1951); O. Jaoul, I. A. Campbell, and A. Fert, J. Magn. Magn. Mater. 5, 23 (1977); A. P. Malozemoff, Phys. Rev. B 32, 6080 (1985).

${ }^{10}$ N. F. Mott, Proc. R. Soc. London, Ser. A 153, 699 (1936).

${ }^{11}$ A. Fert and I. A. Campbell, Phys. Rev. Lett. 21, 1190 (1968).

${ }^{12}$ I. A. Campbell, Phys. Rev. Lett. 24, 269 (1970).

${ }^{13}$ O. Jaoul, I. A. Campbell, and A. Fert, J. Magn. Magn. Mater. 5, 23 (1977).

${ }^{14}$ P. A. Stampe, X. Chen, Z. Wang, H. P. Kunkel, and G. Williams, J. Phys.: Condens. Matter 6, 3045 (1994).

${ }^{15}$ P. A. Stampe, H. P. Kunkel, Z. Wang, and G. Williams, Phys. Rev. B 52, 335 (1995).

${ }^{16}$ J. N. Eckstein, I. Bozovic, J. O'Donnell, M. Onellion, and M. S. Rzchowski, Appl. Phys. Lett. 69, 1312 (1996).

${ }^{17}$ H. S. Wang, Q. Li, K. Liu, and C. L. Chien, Appl. Phys. Lett. 74, 2212 (1999).

${ }^{18}$ J. O'Donnell, M. J. N. Eckstein, and M. S. Rzchowski, Appl. Phys. Lett. 76, 218 (2000).

${ }^{19}$ Q. Li, H. S. Wang, Y. F. Hu, and E. Wertz, J. Appl. Phys. 87, 5573 (2000).

${ }^{20}$ M. Ziese and S. P. Sena, J. Phys.: Condens. Matter 10, 2727 (1998); M. Ziese, Phys. Rev. B 62, 1044 (2000).

${ }^{21}$ B. I. Belevtsev, V. B. Krasovitsky, D. G. Naugle, K. D. D. Rathnayaka, A. Parasiris, S. R. Surthi, R. K. Pandey, and M. A. Rom, Phys. Status Solidi A 188, 1187 (2001).

${ }^{22}$ M. Bibes, V. Laukhin, S. Valencia, B. Martínez, J. Foncuberta, O. Yu Gorbenko, A. R. Kaul, and J. L. Martínez, J. Phys.: Condens. Matter 17, 2733 (2005).

${ }^{23}$ At sufficiently high magnetic fields, the magnetization is aligned with the field orientation, making $\theta \simeq \phi$.

${ }^{24}$ M. Bibes, B. Martínez, J. Foncuberta, V. Trtik, C. Ferrater, and F. Sánchez, J. Magn. Magn. Mater. 211, 206 (2000).

${ }^{25} \mathrm{~A}$ constant resistivity $\left(\approx \rho_{0}\right)$ is subtracted from each as-measured trace to obtain the amplitude of $\rho_{\text {AMR }}$.

${ }^{26}$ Note that Fig. 3(b) only shows data taken for one field direction ( $\theta$ rotated from -90 to $270 \mathrm{deg}$ ).

${ }^{27}$ T. R. McGuire and R. I. Potter, IEEE Trans. Magn. 11, 1018 (1975).

${ }^{28}$ X. W. Li, A. Gupta, G. Xiao, and G. Q. Gong, Appl. Phys. Lett. 71, 1124 (1997).

${ }^{29}$ A. Gupta, G. Q. Gong, G. Xiao, P. R. Duncombe, P. Lecoeur, P. Trouillod, Y. Y. Wang, V. P. Dravid, and J. Z. Sun, Phys. Rev. B 54, R15629 (1996).

${ }^{30}$ A. Biswas, M. Rajeswari, R. C. Srivastava, T. Venkatesan, R. L. Greene, and A. J. Millis, Phys. Rev. B 61, 9665 (2000); A. Biswas, M. Rajeswari, R. C. Srivastava, T. Venkatesan, R. L. Greene, Q. Liu, A. L. de Lozanne, and A. J. Millis, ibid. 63, 184424 (2001).

${ }^{31}$ N. Farag, M. Bobeth, W. Pompe, A. E. Romanov, and J. S. Speck, J. Appl. Phys. 97, 113516 (2005).

${ }^{32}$ A. Khapikov, L. Uspenskaya, I. Bdikin, Ya. Mukovskii, S. Karabashev, D. Shulyaev, and A. Arsenov, Appl. Phys. Lett. 77, 2376 (2000).

${ }^{33}$ J.-L. Maurice, F. Pailloux, A. Barthélémy, O. Durnad, D. Imhoff, R. Lyonnet, A. Rocher, and J.-P. Contour, Philos. Mag. 83, 3201 (2003).

${ }^{34}$ G. H. Jonker and J. H. van Santen, Physica 16, 337 (1950); G. H. Jonker, ibid. 22, 707 (1956); Y. Tokura, A. Urushibara, Y. Moritomo, T. Arima, A. Asamitsu, G. Kido, and N. Furukawa, J. Phys. Soc. Jpn. 63, 3931 (1994); A. Urushibara, Y. Moritomo, T. Arima, A. Asamitsu, G. Kido, and Y. Tokura, Phys. Rev. B 51, 14103 (1995).

${ }^{35}$ Another difference between the uniform and nonuniform states could be in 
the local current direction. While in the uniform state the local and average current directions are the same, in the nonuniform state the local current direction may vary. Further study is required to investigate this effect.

${ }^{36}$ M. Fäth, S. Freisem, A. A. Menovsky, Y. Tomioka, J. Aarts, and J. A. Mydosh, Science 285, 1540 (1999).

${ }^{37}$ A. Urushibara, Y. Moritomo, T. Arima, A. Asamitsu, G. Kido, and Y. Tokura, Phys. Rev. B 51, 14103 (1995); K. Kubo and N. Ohata, J. Phys.
Soc. Jpn. 33, 21 (1972); N. Furukawa, ibid. 63, 3214 (1994).

${ }^{38}$ X. Hong, J.-B. Yau, J. D. Hoffman, C. H. Ahn, Y. Bason, and L. Klein, Phys. Rev. B 74, 174406 (2006).

${ }^{39}$ Note that in manganite thin films with different doping levels the electronic inhomogeneities may affect the local current directions, namely the angle between the local magnetization and the current, which is worth further investigation. 